(2) Open Access Full Text Article

ORIGINAL RESEARCH

\title{
A survey of patient preferences for a placebo orodispersible tablet
}

\author{
This article was published in the following Dove Press journal: \\ Patient Preference and Adherence \\ 19 March 2012 \\ Number of times this article has been viewed
}

\author{
Alan G Wade' \\ Gordon M Crawford' \\ David Young ${ }^{2}$ \\ 'CPS Research, Glasgow, UK; \\ ${ }^{2}$ Department of Mathematics and \\ Statistics, University of Strathclyde, \\ Glasgow, Scotland, UK
}

Correspondence: Alan G Wade CPS Research, 3 Todd Campus, West of Scotland Science Park, Acre Road, Glasgow, G20 0XA, Scotland, UK Tel +44 |4| 9467888

Fax +44 I4I 946 I324

Email alangwade@fastmail.fm
Aim: To assess the attitudes and preferences of patients currently being treated for depression or anxiety disorders with traditional oral antidepressants relative to a placebo orodispersible (ODT) formulation of escitalopram.

Methods: This was an open study collecting patient-reported outcome data from patients with anxiety or depression that were treated with oral antidepressant medication on Day 0 before and after receiving a single placebo ODT, and on Day 3 or 4 after receiving two further daily doses of placebo ODT. Patients aged 18-80 years who were currently receiving treatment with oral antidepressants were recruited from general practice and by advertising. Patients with significant symptoms of anxiety or depression (scoring $\geq 9$ on either the depression or anxiety subscales of the Hospital Anxiety and Depression Scale) were included in the study.

Results: A total of 150 patients were enrolled in and completed the study. About $37 \%$ of the patients had had trouble with swallowing tablets, and patients with higher depression scores reported more general swallowing problems than those with lower scores $(P=0.002)$. Most patients $(75.3 \%)$ believed that an ODT might work faster but that it would make no difference to the effectiveness of the medication $(63.1 \%)$ or the number of side effects $(81.3 \%)$. About $96 \%$ of the patients reported experiencing a pleasant taste following the placebo ODT, although seven patients did not like its taste or aftertaste. This study found that $80.7 \%$ of patients reported that the tablets were easy or very easy to get out of the packaging.

Conclusion: Based on the results of the placebo version of escitalopram ODT, the escitalopram ODT is likely to be well accepted by patients suffering from anxiety or depressive symptoms. Keywords: ODT, swallowing difficulties, antidepressant, depression, anxiety

\section{Introduction}

Escitalopram is a highly selective serotonin reuptake inhibitor (SSRI) used for the treatment of depression and anxiety disorders. It is available as oral coated tablets of 5,10 , and $20 \mathrm{mg}$. A new formulation of an orodispersible tablet (ODT) containing escitalopram has been developed and its pharmacokinetics and pharmacodynamics are similar to that of the existing immediate release tablets. ${ }^{1}$

While obvious benefits for the formulation exist for specific populations such as patients with swallowing difficulties or specific diseases such as Parkinson's, ${ }^{2}$ the benefits for more general groups of patients or for specific subgroups have not been fully evaluated. Although healthy male subjects expressed a preference for ODT escitalopram, ${ }^{1}$ the preferences of patients suffering from depression or anxiety have not been investigated. 
Swallowing problems affecting medication taking and compliance are more common than is often recognized in general practice. ${ }^{3,4}$ A study by Andersen et al found that $26 \%$ of a Norwegian general practice population reported difficulties swallowing tablets, with women having problems twice as frequently as men. ${ }^{5}$ Compliance with medication is a serious problem in patients with depression and may be improved by the use of ODT tablets, which eliminate the need to swallow a tablet. ${ }^{6,7}$ Advantages of the new formulation are also likely to lie in patient attitudes and preferences and in the impact that these have on areas such as stigma, utility, and patient perception that, rightly or wrongly, ODT tablets have improved efficacy and speed of onset.

Studies have been undertaken in focus groups to assess patient preferences for ODT versus oral tablet and the acceptability of the formulation. However, attitudes and preferences are heavily influenced by psychological status and personality traits. Therefore, it was important to assess the new formulation in patients currently being treated for depression or anxiety disorders. The main aim of this open study was to assess the attitudes and preferences of patients currently being treated for depression or anxiety disorders towards a new ODT formulation in comparison to their current treatment with a traditional oral antidepressant. The placebo version of escitalopram ODT was used as the study medication rather than active medication.

\section{Methods}

\section{Study design}

This was an open study collecting patient-reported outcome data from patients with anxiety or depression. Data were collected on Day 0 (both before and after receiving a single placebo ODT) and on Day 3 or 4 after receiving two further daily doses of placebo ODT, so that their use could be assessed in normal circumstances. This study was performed in compliance with good clinical practice. Since this study was designed to assess patient preferences for the ODT, the attitudes and the acceptability of the formulation to patients currently being treated for depression or anxiety disorders was assessed through the use of five patient-reported outcome questionnaires. The placebo version of escitalopram ODT was used as the study medication rather than active medication.

\section{Patients}

Patients aged 18 to 80 years who were currently receiving treatment with oral antidepressant drugs were recruited from general practice and by advertising. These patients were invited to participate in the study and informed consent was obtained. The Hospital Anxiety and Depression Scale $(\mathrm{HADS})^{8}$ was administered to distinguish between patients whose primary symptoms were related to anxiety ( $\geq 9$ on the anxiety subscale, HADS-A) and those whose primary symptoms were related to depression ( $\geq 9$ on the depression subscale, HADS-D). The HADS is a patient-reported scale that includes these two subscales, each of which is composed of seven items that are each rated from 0 to 3 . The patient is required to indicate the response that most accurately reflects the way he/she has felt over the last few days. The scores for questions relating to anxiety and depression are added to obtain the total scores for anxiety and depression. Patients scoring 9 or more on either the depression or anxiety subscales were included in the study. Only patients willing to participate in a questionnaire, take a placebo tablet, and complete a follow-up questionnaire were considered suitable for enrolment. Patient demographic data was collected prior to completing the questionnaires.

\section{Treatment}

The study medication was identical in composition and appearance to escitalopram ODT apart from the lack of active substance, which was replaced by inert filler. The study medication provided by H Lundbeck A/S (Valby, Denmark) was manufactured, packed and shipped according to good manufacturing practice (GMP) and good distribution practice (GDP) standards. Supplies were provided in blister packs of six tablets. Participants were requested to return all unused tablets to the study site for destruction. Patients were administered a single placebo ODT under supervision on Day 0, and the time of the administration was recorded. Each patient was given two ODTs to self-administer as a single tablet on Day 1 and 2. Compliance for this period was recorded during the telephone interview when patients were asked "Did you take both tablets as instructed?"

\section{Patient-reported outcome measurements}

Three pre-dose questionnaires were used during this study: patient attitudes towards their illness and current medication, attitudes to medicines, and a medicine swallowing questionnaire. Immediately after the patient took a single dose of placebo ODT escitalopram, an attitudes/preference questionnaire (post-placebo ODT) was completed. A second questionnaire was conducted by telephone following use of the additional placebo tablets; this assessed any changes that had occurred in the patients' attitudes after they had had additional time to consider the questions, use the ODT tablets, and discuss their use with relatives and friends. 


\section{Statistical methods}

Descriptive statistics were used to summarize the data. The intention to treat (ITT) analysis set, which consisted of all enrolled patients, was used to analyze all patient-reported outcome variables. Kruskal-Wallis tests were used to investigate relationships between age groups and anxiety and depression scores. The association between age groups and responses to the medicines swallowing questionnaire and patient attitude questions were assessed using chi-squared tests. All analyses were done using Minitab (v16; Minitab Inc, State College, PA) at a 5\% significance level.

\section{Results}

\section{Patient disposition}

A total of 150 patients were enrolled in and completed the study. The first patient entered the study in July 2011 and the last patient completed the study in August 2011. The demographic characteristics of study patients are summarized in Table 1.

\section{Baseline characteristics}

The HADS scores of patients are summarized in Table 2. A total of 105 patients ( $70 \%$ of total patients) were categorized as having moderate to severe anxiety $(>11$ on the anxiety subscale), and 77 (51\%) were categorized as having moderate to severe depression $(>11$ on the depression subscale). HADS-A scores of $\geq 9$ were recorded for 135 patients $(90 \%)$ and a HADS-D score of $\geq 9$ was recorded for 110 patients $(73.3 \%)$, with 95 patients $(63.3 \%)$ being anxious and depressed, 40 patients $(26.7 \%)$ being anxious but not depressed, and 15 patients $(10.0 \%)$ being depressed but not anxious. No patients were assessed by the HADS methodology as being both non-anxious and non-depressed.

Table I Demographic characteristics, ITT analysis set $(n=150)$

\begin{tabular}{ll}
\hline & $\mathbf{n}(\%)$ \\
\hline Age (years) & \\
$18-30$ & $16(10.7 \%)$ \\
$31-45$ & $48(32.2 \%)$ \\
$45-60$ & $62(41.6 \%)$ \\
$60-80$ & $23(15.4 \%)$ \\
Missing & $\mathrm{I}$ \\
Gender & \\
Male & $51(34.0 \%)$ \\
Female & $99(66.0 \%)$ \\
Race & \\
Caucasian & $148(99.3 \%)$ \\
Other & $\mathrm{I}(0.7 \%)$ \\
Missing & $\mathrm{I}$ \\
\hline
\end{tabular}

Note: Percentages are expressed as percentage of patients with data.
Table 2 Hospital anxiety and depression (HADS) scale scores, ITT analysis set $(n=150)$

\begin{tabular}{ll}
\hline Parameter & \\
\hline Anxiety: total HADS-A score & \\
Median & 13 \\
Range & $3-21$ \\
QI, Q3 & 10,15 \\
Category, $n(\%)$ & \\
Abnormal & $105(70 \%)$ \\
Borderline normal & $33(22 \%)$ \\
Normal & $12(8 \%)$ \\
Depression: total HADS-D score & \\
Median & 11 \\
Range & $1-14$ \\
QI, Q3 & 8,14 \\
Category, n (\%) & \\
Abnormal & $77(51.3 \%)$ \\
Borderline normal & $44(29.3 \%)$ \\
Normal & $29(19.3 \%)$ \\
\hline
\end{tabular}

Notes: Categories of anxiety and depression levels were defined based on HADS scale scores in which $0-7=$ normal, $8-10=$ borderline abnormal, and ||$-2 \mid=$ abnormal.

Abbreviations: HADS-A, hospital anxiety and depression scale (anxiety); HADS-D, hospital anxiety and depression scale (depression); ITT, intention to treat.

All 150 patients were taking antidepressant medication upon enrolment into the study. The most commonly prescribed antidepressant medications were citalopram (33.3\%) and fluoxetine $(22.7 \%)$. In addition, three patients (2\%) were recorded as taking anxiolytics; two patients (1.3\%) took diazepam, and one patient $(0.7 \%)$ took buspirone. Most patients $(76 \%)$ had been taking their antidepressant medication for more than 6 months. Concomitant medication taken after study entry was not recorded.

Treatment compliance was $100 \%$ for the single ODT administered in the clinic under supervision. Compliance was also $100 \%$ for days 1 and 2, as assessed from the responses given in the post-interview telephone questionnaire.

\section{Patient reported outcomes}

\section{Tablet swallowing questionnaire}

While 61 patients $(40.7 \%)$ in this study reported no problems swallowing tablets, 56 patients $(37.3 \%)$ had problems swallowing medications, and a total of 115 patients $(76.7 \%)$ indicated a preference for a tablet that was easy to swallow. Patients had previously used several methods to make a tablet easier to swallow, with $22.7 \%$ having crushed the tablet, $7.3 \%$ having emptied the contents of a capsule, $8.7 \%$ having tried to dissolve medication in water, and $24.7 \%$ having used another method, usually dividing or breaking the tablet.

\section{Patient attitude on illness and current medication}

Patients were aware of the mental illnesses they were suffering from and most reported that they suffered from 
more than one condition. Moderate to severe depression (reported in 59.3\% of patients) and anxiety (56.0\%) were the most commonly reported conditions, with $30 \%$ reporting stress and $18.7 \%$ reporting bereavement reaction. The majority of patients ( $86 \%$ ) had long-standing illness (more than 1 year) and $64.4 \%$ had been taking antidepressant medication for more than one year. Most patients (94\%) had told family or friends about their illness and $71.3 \%$ reported that they were getting at least a fair amount of support from them.

Morning was the most commonly reported time for taking antidepressant medication in this study with $58.7 \%$ of patients taking their tablets at this time. Their reported compliance was high with $44 \%$ stating that they never missed a tablet and $54 \%$ skipping a tablet only occasionally. The main reasons reported to potentially make patients less likely to regularly take their treatment were side effects $(36 \%)$ and uncertainty regarding efficacy $(17.3 \%)$. There were 60 (40.3\%) patients who had missed work or daily activities for $>5$ days due to their illness in the previous month, but 51 (34.2\%) who had not missed a day.

\section{Attitudes to medicines - pre-dose}

Patients were asked to rank the importance of nine tablet characteristics on a scale from 1 (very important) to 9 (not important at all). There was a significant difference between the nine ideal tablet characteristics (Kruskal-Wallis test, $P<0.001$ ). The most important ideal characteristics (all with a median score of 1 ) were the need to take a tablet less frequently, that it was easy to swallow, that it was effective at treating the illness, that it have no serious side effects even if they are rare, that it have no side effects at all, and that it relieved symptoms quickly. The lowest ranking was for 'the tablet feels and looks new and modern' (median score of 9). A pleasant taste (median score of 3 ) and ease of getting out of the packaging (median score of 2) were also rated less important than other characteristics.

Thirty-five patients $(23.5 \%)$ had previously taken an orodispersible tablet. About half (50.7\%) of all patients thought that a tablet that melted in the mouth would make it easier to take medication regularly. The convenience of being able to take a tablet while out of the house, the fact that it does not need to be swallowed, and that it could be taken without work colleagues/acquaintances seeing them taking it were perceived as advantages of an orodispersible antidepressant tablet by $93.8 \%, 79.1 \%$, and $72.3 \%$ of patients, respectively. Most patients $(75.3 \%)$ believed that an ODT might work faster but that it would make no difference to the effectiveness of the medication $(63.1 \%)$ or the number of side effects experienced $(81.3 \%)$.

\section{Attitudes to medicines - post placebo ODT}

A high proportion (96\%) of the patients reported experiencing a pleasant taste following the placebo ODT and $80.7 \%$ found that the tablets were easy or very easy to get out of the packaging. No patient had any difficulty with picking up the tablet and placing it in the mouth, with $77.3 \%$ of patients finding it a very easy process. Having taken an ODT placebo, $81.9 \%$ of patients thought that a real ODT tablet would work more quickly than their existing tablet and $81.3 \%$ expressed a preference for this type of tablet. Of the 126 patients (84\%) who expressed a definite preference ("yes", 95 patients; "no", 31 patients) for this type of tablet, a significantly higher proportion said they would be more likely to take this type of tablet every day $(75.4 \%$ versus $24.6 \%$, Z-test, $P<0.001)$. Four patients commented that they would have preferred/suggested another flavor and three patients found that the tablet had a chalky or powdery taste.

\section{Post-interview telephone questionnaire}

Most patients (96\%) had not changed their attitude to discussing their illness with family and friends or taking their medication regularly since the post-placebo interview, and the taste responses given in the post-interview telephone questionnaire were similar to those obtained in the postplacebo questionnaire. Thirty-five patients $(23.3 \%)$ found it slightly difficult or very difficult to get the tablets out of the packaging compared with 25 patients (16.7\%) after the first dose. Responses to the question "Would you prefer your tablets to be like this?" were comparable with those obtained after single dose, although there was an increase in the number of patients preferring to stay on their existing medication (increased from six to 13 patients), and fewer patients responded that they would be less likely to take this type of tablet every day (decreased from 31 patients to 21 patients).

\section{Additional statistical analyses}

There were no clear associations between age and the responses to the medicine swallowing questions, although there was evidence of an association between depression and swallowing problems (Pearson $\chi^{2}$ statistic of 9.171, $P=0.002)$. Patients with depression scores $\geq 9$ were more likely to report having swallowing problems swallowing food than those with depression scores $<9$. 


\section{Discussion}

All patients in this open study were taking antidepressant medication and the majority had been doing so for more than a year. The severity of reported symptoms in patients who were predominantly on long-term antidepressant treatment may indicate a selected population who volunteered for this study, nevertheless it reflects the high symptomatology of patients on long-term antidepressant therapy previously reported in an earlier "well-being" study. ${ }^{9}$ However, the study population is considered to be a representative sample of the intended target population. The sample size of 150 patients was also considered to be sufficient to obtain meaningful descriptive data.

Regan et al reported a higher proportion of patients with severe mental health problems complaining of swallowing difficulties than would be expected in the general population. ${ }^{10}$ The current study also found evidence of an association between depression severity and swallowing problems, with patients with higher depression scores reporting more general swallowing difficulties than those with lower scores. Thirty-seven percent of the patients in the current study had trouble swallowing tablets and more than one-fifth of patients reported that they had at some time crushed their tablets or emptied out the capsule before taking it. While these figures may appear high, they accord well with those reported by Llorca $^{4}$ and the fact that over one-fifth of patients had taken measures to circumvent the problem suggests that swallowing problems may be significant. Furthermore, the current study suggests that ease of swallowing is an important characteristic in an ideal tablet, with most patients indicating a definite preference for a tablet that was easy to swallow.

Compliance with medication is a significant problem in most chronic diseases and particularly so for depression. The impact of the formulation of the medication on compliance cannot be determined with confidence from this study but a significantly higher proportion of patients thought that a tablet that melted in the mouth would make it easier to take regularly than those who thought that it would not make a difference, which is in accord with results reported by Roose ${ }^{11}$ and Benkert et al ${ }^{12}$ for mirtazapine. Overall, the results demonstrate that there is a real need for an antidepressant tablet that is easy to take.

The general ease of use of an orodispersible antidepressant tablet was further demonstrated by the patients' reporting that these tablets are preferred as they do not need to be swallowed, are convenient to take when out of the house, and can be taken without work colleagues/acquaintances seeing them doing so. Side effects are one of the main reasons that patients may be less likely to take their treatment regularly, and the patients in the current study felt that an ideal tablet would have no serious side effects even if they are rare, or have no side effects at all. However, patients were realistic in their expectations of orodispersible tablets. Prior to taking a placebo ODT, most patients did not expect this type of tablet to affect the incidence of side effects or the effectiveness of the medication, but believed that an antidepressant orodispersible tablet might work faster.

Morning was the most commonly reported time for taking antidepressant medication. Having taken a placebo ODT, most patients thought that a real (active escitalopram) tablet would work more quickly than their existing tablet and expressed a preference for this type of tablet. The process of getting the tablets out of the packaging was an easy task for the vast majority of patients. Patients were also likely to enjoy the experience of taking the tablets as almost all patients thought that the placebo version of escitalopram ODT had a pleasant taste, although a few did not like its taste or aftertaste.

Patients generally expressed similar attitudes and preferences before and after taking the placebo version of the ODT and also after having had time to reflect on their thoughts after taking two further doses at home. However, there was some evidence that more patients found it slightly difficult or very difficult to get the tablets out of the packaging in the home environment than they did after taking the first dose under supervision.

There were limitations with the current study. The patients were recruited through general practitioner referral and advertising, and there may be some recruitment bias with motivated patients more likely to respond to these methods of recruitment. In addition, the patients were asked to complete five patient-reported outcome questionnaires with a total of 52 questions, and they may not be fully focused on correctly and honestly answering such a large number of questions. The placebo version of escitalopram was used as the study medication and there may be problems with extrapolating some of the results such as taste perception to the active escitalopram ODT.

\section{Conclusion}

The majority of patients in this study had long-standing anxiety or depression and they had been taking antidepressant medication for more than 1 year. Their reported compliance with taking antidepressant medication was high. Family or friends were generally aware of their illness and the majority of patients were getting some support from them. 
Many patients had problems swallowing medications and most reported that they would prefer to use a tablet that is easy to swallow. Patients with higher depression scores may have more general swallowing problems than those with lower depression scores.

The escitalopram ODT is likely to be well accepted by patients suffering from anxiety or depressive symptoms based on the results of the placebo version used in the current study. Most patients reported that the placebo version of escitalopram ODT had a pleasant taste, although a few did not like the taste or the aftertaste of the product. Patients generally preferred this type of tablet to the traditional formulations and felt that they would be more likely to take it regularly. Overall, they expected an active ODT to work more quickly than their existing tablet but did not expect an ODT to make any difference to the incidence of side effects.

\section{Acknowledgments}

The authors thank the patients who participated in the study and the staff of CPS Research for their dedication to the recruitment and management of the patients and the timely completion of the study.

\section{Disclosure}

This research was sponsored by an unrestricted grant from H Lundbeck A/S, Copenhagen, Denmark.

\section{References}

1. Nilausen DØ, Zuiker R, van Gerven J. The perception and pharmacokinetics of a $20-\mathrm{mg}$ dose of escitalopram orodispersible tablets in a relative bioavailability study in healthy men. Clin Ther. 2011;33(10):1492-1502.

2. Hunter PC, Crameri J, Austin S, Woodward MC, Hughes AJ. Response of parkinsonian swallowing dysfunction to dopaminergic stimulation. J Neurol Neurosurg Psychiatry. 1997;63(5):579-583.

3. Navarro V. Improving medication compliance in patients with depression: Use of orodispersible tablets. Adv Ther. 2010;27(11):785-795.

4. Llorca P-M. Discussion of prevalence and management of discomfort when swallowing pills: orodispersible tablets expand treatment options in patients with depression. Ther Deliv. 2011;2(5):611-622.

5. Andersen O, Zweidorff OK, Hjelde T, Rødland EA. Problems when swallowing tablets. A questionnaire study from general practice. Tidsskr Nor Laegeforen. 1995;115(8):947-949. Norwegian.

6. Márquez-Contreras E, Gil V, López J, et al. Pharmacological compliance and acceptability of lansoprazole orally disintegrating tablets in primary care. Curr Med Res Opin. 2008;24(2):569-576.

7. Koh N, Sakamoto S, Chino F. Improvement in medication compliance and glycemic control with voglibose oral disintegrating tablet. Tohoku J Exp Med. 2008;216(3):249-257.

8. Zigmond AS, Snaith RP. The hospital anxiety and depression scale. Acta Psychiatr Scand. 1983;67:361-370.

9. Wade AG, Crawford GM, Bezlyak V. Wellbeing of patients prescribed long-term SSRIs by general practitioners in the UK [abstract]. Eur Neuropsychopharmacol. 2008;18 Suppl 4:S333.

10. Regan J, Sowman R, Walsh I. Prevalence of dysphagia in acute and community mental health settings. Dysphagia. 2006;21(2):95-101.

11. Roose SP. Compliance: the impact of adverse events and tolerability on the physician's treatment decisions. Eur Neuropsychopharmacol. 2003;13 Suppl 3:S85-S92.

12. Benkert O, Szegedi A, Philipp M, et al. Mirtazapine orally disintegrating tablets versus venlafaxine extended release: a double-blind, randomized multicenter trial comparing the onset of antidepressant response in patients with major depressive disorder. J Clin Psychopharmacol. 2006;26:75-78.
Patient Preference and Adherence

\section{Publish your work in this journal}

Patient Preference and Adherence is an international, peer-reviewed, open access journal focusing on the growing importance of patient preference and adherence throughout the therapeutic continuum. Patient satisfaction, acceptability, quality of life, compliance, persistence and their role in developing new therapeutic modalities and compounds to

\section{Dovepress}

optimize clinical outcomes for existing disease states are major areas of interest. This journal has been accepted for indexing on PubMed Central. The manuscript management system is completely online and includes a very quick and fair peer-review system. Visit http://www.dovepress.com/ testimonials.php to read real quotes from published authors. 